\title{
A Fluctuation-Dissipation Model for Electrical Noise*
}

\author{
Jose-Ignacio Izpura, Javier Malo \\ Group of Microsystems and Electronic Materials, Universidad Politécnica de Madrid, Madrid, Spain \\ E-mail: joseignacio.izpura@upm.es \\ Received February 5, 2011; revised April 6, 2011; accepted April 13, 2011
}

\begin{abstract}
This paper shows that today's modelling of electrical noise as coming from noisy resistances is a non sense one contradicting their nature as systems bearing an electrical noise. We present a new model for electrical noise that including Johnson and Nyquist work also agrees with the Quantum Mechanical description of noisy systems done by Callen and Welton, where electrical energy fluctuates and is dissipated with time. By the two currents the Admittance function links in frequency domain with their common voltage, this new model shows the connection Cause-Effect that exists between Fluctuation and Dissipation of energy in time domain. In spite of its radical departure from today's belief on electrical noise in resistors, this Complex model for electrical noise is obtained from Nyquist result by basic concepts of Circuit Theory and Thermodynamics that also apply to capacitors and inductors.
\end{abstract}

Keywords: Admittance-Based, Noise Model, Fluctuation, Susceptance, Dissipation, Resistance

\section{Introduction}

Current understanding of electrical noise in circuits considers shot and Johnson noises as different physical phenomena each with its own physical model, despite their deep connection found by some authors [1]. Considering electric current as carried by discrete electrons independently of one another, as Johnson did in vacuum devices to study their shot noise [2], this connection isn't too surprising. What is a surprise, however, is that today's works in this field tend to consider noise currents as carried by packets of electrons that, to our knowledge, hardly are found in ordinary matter. We mean proposals like [3] contending that electrical charge piling-up in a lonely resistance $R$ generates shot noise, thus transgressing a Quantum-Mechanical result of [4]: the need for a Complex Admittance function to describe a noisy system.

This transgression and that of Special Relativity that a null $C$ shunting the $R$ of a resistor endures [5], led us to review today's modelling of electrical noise in this paper showing why the PSPICE simulator does not give the right noise of junction diodes [6] and what the effects are of today's unawareness about the Susceptance that shunts the Conductance $G=1 / R$ of any resistor we can make. The paper is organized as follows. In Section 2 we

*Work supported by the Spanish CICYT under the MAT2010-18933 project, by the Comunidad Autónoma de Madrid through its IV-PRICIT Program, and by the European Regional Development Fund (FEDER). review the Partial Interpretation (PI) in use today of Johnson-Nyquist results that leads to a wrong modelling of electrical noise sometimes. Section 3 shows how to pass from this PI to an Advanced Model (AM) for electrical noise that agreeing with the laws of Physics, also allows a right modelling of this noise where the aforesaid PI fails. This passage is done in frequency domain by the familiar noise densities $S_{I}(f)$ in $\mathrm{A}^{2} / \mathrm{Hz}$ and $S_{V}(f)$ in $\mathrm{V}^{2} / \mathrm{Hz}$. Section 4 , however, considers the generation of electrical noise by Fluctuations and Dissipations of electrical energy in time domain, taking place in the Admittance of two-terminal devices like resistors and capacitors, no matter its physical structure. Some relevant conclusions are drawn at the end.

\section{Reviewing Current Model for Electrical Noise in Resistors}

To a first level, the Susceptance shunting the conductance $G=1 / R$ of a resistor of resistance $R$ is due to a capacitance $C_{d}$ coming from the dielectric properties of the material used to fabricate this device, whose physical structure appears in Figure 1 and where the space between two parallel plates (ohmic contacts) contains some material of dielectric permittivity $\varepsilon$ and conductivity $\sigma$. We mean the Susceptance due to $C_{d}=\tau_{d} / R$ that each resistor bears in parallel with its $R$ because the non-null dielectric relaxation time $\tau_{d}=\varepsilon / \sigma$ of its inner material 
[7]. Any stray capacitance as $C_{\text {stray }} \approx 0.5 \mathrm{pF}$, typical of set-ups used in Low Frequency Noise (LFN) measurements [8], would be added to $C_{d}$.

For conducting materials as n-doped Silicon or GaAs, $\tau_{d}$ falls below the ps, thus giving very low $C_{d}$ values: $C_{d} \approx 10^{-15} \mathrm{~F}$ for $R=1 \mathrm{k} \Omega$ and $\tau_{d}=1 \mathrm{ps}$. This is why $C_{d}$ and $C_{\text {stray }}$ are considered irrelevant at frequencies $f \leq 1$ $\mathrm{MHz}$ where LFN is found. This irrelevance that can be accepted numerically in most cases (e.g. when there are other Admittances in parallel), leads to the misconception underlying the aforesaid PI of the pioneering works of Johnson [9] and Nyquist [10]. This error is so deep-rooted in today's research that reviewers of [3] were not ashamed of publishing a piling-up of electrical charge in a resistance devoid of capacitance $C$, which links electric charge $Q(t)$ and electric voltage $v(t)$. When a current $i(t)$ produces a change of dipolar charge $\partial Q=i \partial t$ as $t$ passes, it builds a voltage $\partial v$ that is linked with the charge variation at each instant by the Capacitance $C=\partial Q / \partial v$. Thus, $C=0$ between the two terminals of a resistor means that it could show an electrical voltage between them without electric charges acting as its source or, as we wrote in [5], its material having finite $\sigma$ to offer a finite $R$ with the structure of Figure 1, but having a null permittivity $\varepsilon=0$ to offer $C_{d}$ $=0$, would allow an infinite speed for the electromagnetic wave, thus infringing Einstein's Special Relativity. Besides this conflict with Physics, this PI of Nyquist work is hard to apply out of Thermal Equilibrium (TE) because [10] was done for TE. The footnote of Figure 4.11 of [11] summarizes the essence of this PI by this sentence: "Circuits elements and their noise models. Note that capacitors and inductors do not generate noise".

Figure 2 shows the circuits this PI uses to represent electrical noise in a resistor of resistance $R$, where a Conductance $G=1 / R$ is shunted by a noise generator of density $S_{I}(f)=4 k T / R \quad \mathrm{~A}^{2} / \mathrm{Hz}$ (Nyquist noise) for $k$ being the Boltzmann constant. This leads to a noise density $S_{V}(f)=R^{2} \times S_{I}(f)=4 k T R \quad \mathrm{~V}^{2} / \mathrm{Hz} \quad$ (Johnson noise). Thus, a resistor is a noisy resistance [3] for this PI that uses the resistance $R$ of a resistor in TE as a generator of flat $S_{I}(f)$ and $S_{V}(f)$ noises up to frequencies $f_{Q} \approx 6.2 \mathrm{THz}$ at room $T$, a quantum limit given in [10].

Let's consider under this PI the circuit of Figure 3 with $C=0$, where the voltage of a resistor of $R=100 \mathrm{k} \Omega$ is sampled and feedback as a current by a feedback factor $\beta=1 / R \mathrm{~A} / \mathrm{V}$. For this $R=100 \mathrm{k} \Omega$ giving $S_{V}(f)=4 k T R$ $\approx 1600(\mathrm{nV})^{2} / \mathrm{Hz}$ and $S_{I}(f)=0.16(\mathrm{pA})^{2} / \mathrm{Hz}$, the noiseless sampling and feedback of Figure 3 could be carried out quite well with today's Operational Amplifiers [12] having input noise parameters as: $e_{n}^{2}=36(\mathrm{nV})^{2} / \mathrm{Hz}$ and $i_{n}^{2}=1(\mathrm{fA})^{2} / \mathrm{Hz}$. Accordingly to the PI in use today this negative feedback adds a noiseless $R$ in parallel with the noisy $R$ or resistor whose Nyquist noise $S_{I}(f)$ is the input signal of this feedback circuit. Thus, the $S_{I}(f)$ of the noisy $R$ is the only source of noise in this circuit that, attenuated by the noiseless $R$, leads to $S_{V F B}(f)=k T R \quad \mathrm{~V}^{2} / \mathrm{Hz}$ in it. This result agreeing with

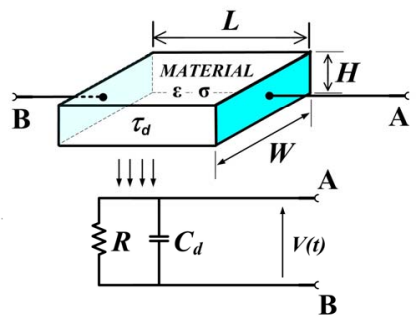

Figure 1. Physical structure of an elemental resistor whose ohmic contacts (shaded area) are the plates that enclose the parallel-plate capacitor of capacitance $C_{d}=\tau_{d} / R$ that allows to apply or to measure an electrical voltage between the terminals $A$ and $B$ of its resistance $R$.

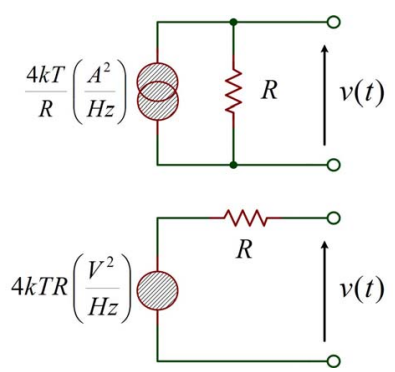

Figure 2. Parallel and series circuits that today's Partial Interpretation (PI) of Johnson and Nyquist results uses to represent the electrical noise of a resistor (see text).

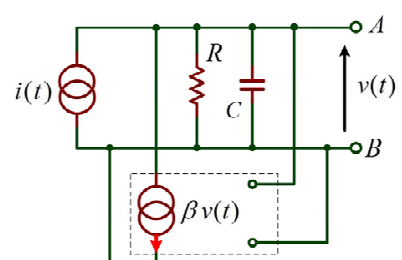

(a)

For $\beta=1 / R$ we have:

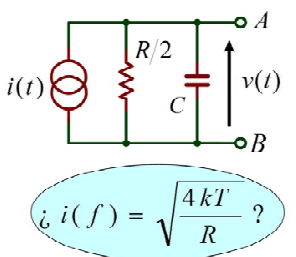

(b)

Figure 3. Feedback scheme used to show that today's unawareness about the role of Susceptance in electrical noise, doesn't allow finding the "well known" $k T / C$ noise of the capacitor existing in this circuit. 
a "well know" one of this PI, namely: "the available noise power from a resistor of resistance $R$ in $T E$ at some $T$ is $N(f)=k T \quad \mathrm{~W} / \mathrm{Hz}$ ", deserves some attention. Because the noisy $R$ and the noiseless $R$ are driven by $S_{V F B}(f)=k T R \quad \mathrm{~V}^{2} / \mathrm{Hz}$, they are dissipating $N(f)=$ $S_{V F B}(f) / R=k T \quad \mathrm{~W} / \mathrm{Hz}$, of course. Since the noisy $R$ is out of $T E$ due to its feedback, we find logical this lower dissipation than the $4 k T \mathrm{~W} / \mathrm{Hz}$ it dissipated in TE when it was driven by $S_{I}(f)$. What is less "logical" is the null dissipation $R$ has in the equivalent circuit of Figure 2 with $S_{V}(f)$ series connected with $R$, thus raising doubts about the physical correctness of $S_{V}(f)=$ $R^{2} \times S_{I}(f)$, whose circuital correctness is well known.

To make some progress, let's consider the noisy $R$ shunted by $C=1 \mathrm{pF}$ in Figure 3 to represent a pixel of an imaging device becoming charged by $m$ electrons when a short packet of $m / \eta$ photons is absorbed with quantum efficiency $\eta<1$. "Short" means that photons are absorbed within a time interval $\Delta t \ll \tau=R C$, where $\tau$ is the time constant of this $R-C$ cell. Thus, a photon packet sets a charge of $m q \mathrm{C}$ in $C$ ( $q$ is the electron charge) and the voltage $\Delta v=m q / C \mathrm{~V}$ appearing on $R$ will be the aimed Signal on $R$ whose power is: $S=$ $(m q / C)^{2} / R$ W. Since $C$ will discharge through $R$, the Signal readout would be done a short time $t_{d} \ll \tau$ after the photon absorption in order to find the aforesaid power $S$. Concerning Noise power $N$, the mean square noise voltage on $R$ is the product of the Johnson noise $S_{V}(f)=4 k T R \quad \mathrm{~V}^{2} / \mathrm{Hz}$ by the noise bandwidth $B W_{N}=$ $(\pi / 2) f_{C}$ of this circuit of cut-off frequency $f_{C}=1 /(2 \pi R C)$ [13]: $N=S_{V} \times B W_{N}$. This replaces the integration in $f$ of $S_{V}(f)$ to allow a fast reasoning using Signal to Noise ratios $(S / N)$. Thus, the noise power in the pixel without feedback is: $N=k T /(R C) \mathrm{W}$ and its $S / N$ is: $(S / N)_{\mathrm{i}}=(m q)^{2} /(k T C)$, the ratio of the square charge signal in $C(m q)^{2} \mathrm{C}^{2}$, divided by the square charge noise of $C: k T C \mathrm{C}^{2}$ [14]. To give some figures, the $k T / C$ noise at room $T$ (e.g. the square root of $k T / C$ ) of $C=1$ $\mathrm{pF}$ is $64 \mu \mathrm{V}_{\mathrm{rms}}$. For a Signal $\Delta v=\mathrm{mq} / C=6.4 \mathrm{mV}$ we would have: $S /\left.N\right|_{\mathrm{i}}=10^{4}$ or $40 \mathrm{~dB}$ for the pixel without feedback.

With feedback, however, the $B W_{N}$ doubles due to $\mathrm{R} / 2$ shunting the same $C$ that this feedback in-phase with the output doesn't vary [15]. From this double $B W_{N}$ with $S_{V F B}(f)=k T R$ the noise power on $R$ is: $N_{F B}=k T /(2 R C) \mathrm{W}$. Since the Signal power on $R$ doesn't change because $C$ doesn't vary, the $(S / N)$ of the pixel with feedback would be doubled, a result well worth patenting provided it was true. Unfortunately, this noise modelling is wrong as we will show in the next Section.

\section{An Advanced Model for Electrical Noise in Resistors and Capacitors}

Because $C=\partial Q / \partial v$ links charge variations with voltage ones related with electric fields storing energy between terminals, $C$ behaves as the reservoir of electrical energy whose thermal Fluctuations are observed as Johnson noise $v(t)$ in resistors [7]. Using Thermodynamics, $C$ sets the Degree of Freedom to store electrical energy linked with the fluctuating $v(t)$ in a resistor that a lonely resistance doesn't have. Using Quantum Mechanics we will say that eingenstates of electrical energy hardly will be found in a lonely resistance unable to store it by some Susceptance in parallel or by a Reactance in series. Thus, a cogent model for electrical noise in resistors must handle Conductance $G=1 / R$ together with Susceptance, and this also holds for noise modelling of capacitors in TE at some temperature $T$ because they offer a similar Admittance [5].

It is worth noting that these ideas about noise in resistors are present in Figure 2 if one knows how to read its first circuit or which is the physical meaning of $S_{I}(f)=$ $4 k T G \quad \mathrm{~A}^{2} / \mathrm{Hz}$, a current-like power source proportional to the conductance it is driving. This $S_{I}(f)$ leads to $S_{V}(f)=4 k T R \quad \mathrm{~V}^{2} / \mathrm{Hz}$, a power density that, being proportional to $R$ while its bandwidth (note that $C_{d}$ always exists) is proportional to $1 / R$, means that the mean square voltage noise has nothing to do with currents in $R$. Thus, the noise currents that $S_{I}(f)=4 k T / R$ represents must be currents $i_{n Q}(t)$ orthogonal to noise currents $i_{n P}(t)$ in $G$ that always are in-phase with the voltage noise $v_{n}(t)$. This means currents $i_{n Q}(t)$ always in quadrature with $v_{n}(t)$ flowing through a Susceptance shunting $G=1 / R$. The compound device Nyquist used in [10] agrees with this feature of the AM for electrical noise we are going to show. This AM used to explain the $1 / f$ excess noise of Solid-State devices [7] and the flicker noise of electron fluxes in vacuum ones [5], never has been described before at this level of detail, especially in time domain.

The Susceptance or ability to store electrical energy our AM uses replacing the Transmission Line of [10], is the circuit element providing a Degree of Freedom to store electrical energy liable to fluctuate thermally in the device. These Fluctuations giving rise to the Johnson noise of resistors, don't take place in $R$, but in its Susceptance that must be capacitive because the flat $S_{V}(f)=4 k T R \quad \mathrm{~V}^{2} / \mathrm{Hz}$ found by Johnson at low $f$ [9] is not possible for an inductive Susceptance with Fluctuations of energy of finite power. To make easier the passage from the PI to our AM let's consider the electrical noise of a two-terminal circuit that can be reduced to a capacitance $C$ at high $f$. The equipartition value for one 
Degree of Freedom applies to $C$ in this case [14] and this gives the mean square voltage noise of $C$ in TE from this equality:

$$
\frac{1}{2} k T=\frac{1}{2} C\left\langle v^{2}(t)\right\rangle \Rightarrow\left\langle v^{2}(t)\right\rangle=\frac{k T}{C}
$$

thus showing that the mean square voltage of noise has nothing to do with currents in $R$ as we concluded previously from Circuit Theory and why this is so.

Because (1) gives the $k T / C$ noise of capacitive devices that doesn't depend on the $R$ that shunts $C[13,14]$, let us consider the noise of the BAT85 Schottky diode of [6] whose PSPICE model appears in Appendix I. Due to its $C_{J 0}=11.1 \mathrm{pF}$, the $k T / C$ noise of this diode at room $T$ is: $\left\langle v^{2}(t)\right\rangle=3.7 \times 10^{-10} \mathrm{~V}^{2}$ or $19.3 \mu \mathrm{V}_{\text {rms. }}$. From its saturation current $I_{\text {sat }}=211.7 \mathrm{nA}$ and the ideality factor $n$ $=1.016$ of its $i-v$ curve, $C_{J 0}$ is shunted in TE by a resistance $\left.r_{d 0=}(\partial i / \partial v)^{-1}\right|_{v=0}=n V_{T} / I_{\text {sat }}=124.1 \mathrm{k} \Omega$, where $V_{T}=k T / q$ is the thermal voltage. Following our AM, the room $T$ noise density of this diode at low $f$ must be: $S_{V A M}(0)=4 k T r_{d} \quad \mathrm{~V}^{2} / \mathrm{Hz}$ in TE because this is the value giving $\left\langle v^{2}(t)\right\rangle^{d}=k T / C_{J 0}$ in $C_{J 0}$ to fulfil (1). Therefore, $S_{V A M}(f)$ is this low-pass, Lorentzian spectrum:

$$
S_{V A M}(f)=\frac{4 k \operatorname{Tr}_{d 0}}{1+\left(2 \pi f r_{d 0} C_{J 0}\right)^{2}}=\frac{k T}{C_{J 0}} \times \frac{2}{\pi} \times \frac{\frac{1}{f_{c}}}{1+\left(\frac{1}{f_{c}}\right)^{2}}
$$

that integrated from $f \rightarrow 0$ to $f \rightarrow \infty$ gives $\left\langle v^{2}(t)\right\rangle$ $=k T / C_{J 0}$ as it must be by Equipartition.

Using PSPICE we have obtained the $i-v$ curve of this diode shown in Figure $\mathbf{4}$ with a logarithmic axis to show its exponential character. Besides the simulated $i-v$ we also present (by dots) the $i-v$ solely due to thermoionic current represented by this equation:

$$
\begin{aligned}
i & =I_{\text {sat }}\left(\exp \left(\frac{v}{n V_{T}}\right)-1\right) \\
& =211.7 \cdot 10^{-9}\left(\exp \left(\frac{v}{1.016 \times 25.85 \times 10^{-3}}\right)-1\right)
\end{aligned}
$$

The superposition of the two curves shows that currents through $R_{1}=36 \mathrm{M} \Omega$ used by PSPICE to get a better fitting in reverse mode (see Appendix I), are negligible. The inset of Figure 4 shows the $i$ - $v$ curve with linear axis in the region $(v \approx 0)$ where a resistance $R^{*}(v=0)=123.7$ $\mathrm{k} \Omega$ can be found. This $R^{*}$ is: $R^{*}=\left(r_{d 0} \times R_{1}\right) /\left(r_{d 0}+R_{1}\right) \approx r_{d 0}$. Curve a) of Figure 5 is the $S_{V}(f)$ PSPICE gives for this diode in TE ( $i=0$ in the inset of Figure 5), whose flat $S_{V P I}(0)=-171.5 \mathrm{~dB}$ at low $f$ means $1.13 \mu \mathrm{V}_{\text {rms }}$ noise in this diode whereas our AM states that it will be 19.3 $\mu \mathrm{V}_{\text {rms. }}$. This $S_{V P I}(0)$ being $24.6 \mathrm{~dB}$ below $S_{V A M}(0)=4 k T R^{*}$
$=-146.9 \mathrm{~dB}$, is a 292 times lower noise that PSPICE gives because it considers (as the aforesaid PI) that the only source of noise in the diode at $v=0$ is $R_{1}=36 \mathrm{M} \Omega$, whose Nyquist noise $S_{I}(f)=4 k T / R_{1}$ drives the parallel circuit of $r_{d 0}, R_{l}$ and $C_{J 0}$. This gives a low-pass spectrum of amplitude $S_{V P I}(0)=4 k T\left(R^{*}\right)^{2} / R_{1} \mathrm{~V}^{2} / \mathrm{Hz}$ at low $f$ that is $R_{I} / R^{*}$ times lower than the $S_{V A M}(0)=4 k T R^{*}$ that our AM states to keep the $k T / C_{J 0}$ noise of $C_{J 0}$ shunted by $R^{*}$. Since $10 \log \left(R_{1} / R^{*}\right)=24.6 \mathrm{~dB}$, this explains why today's $P I$ of Nyquist result fails to give a well known $k T / C$ noise. Concerning circuit elements, PSPICE consider them perfectly as it is shown by the cut-off frequency $f_{c}=1 /\left(2 \pi R^{*} C_{J 0}\right)=115.6 \mathrm{kHz}$ of curve a in Figure 5.

Taking advantage of the correctness of the $f_{c}$ PSPICE gives, we have simulated this diode out of TE, under a forward current $i=I_{\text {sat }}$. The noise spectrum thus obtained is curve b of Figure 5, whose $f_{c}=222.4 \mathrm{kHz}$ comes from the low dynamical resistance of the diode under this forward bias: $r_{d}\left(i=I_{s a t}\right)=r_{d f w}=62 \mathrm{k} \Omega$, and its similar junction capacitance (slightly higher with the voltage $v=$ $18.2 \mathrm{mV}$ set by this $i=I_{\text {sat }}$ biasing the diode). Note that while the diode capacitance varies slightly for such a small voltage, the diode resistance varies as: $\exp \left(-v / n V_{T}\right)$

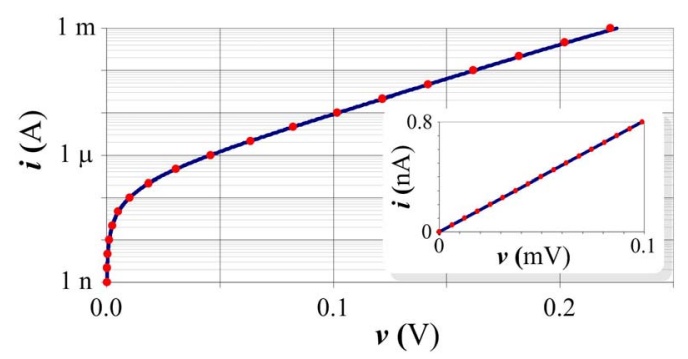

Figure 4. Current-voltage (i-v) characteristics of the BAT85 Schottky diode obtained by PSPICE (line) and its thermoionic values (dots) given by (3) (see text).

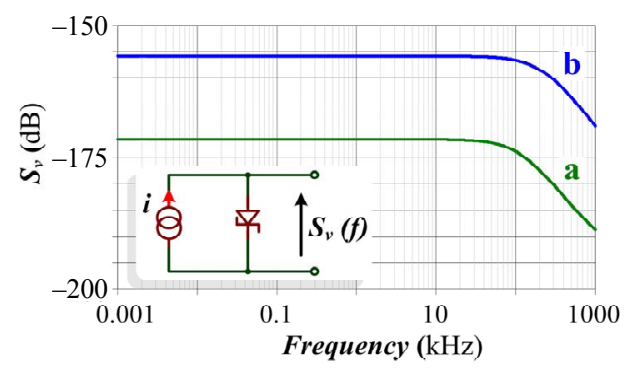

Figure 5. Noise densities $S_{V}(f)$ obtained by PSPICE for the BAT85 Schottky diode at $\operatorname{room} T$ : a) without bias (Thermal Equilibrium, $i=0$ ) and b) under forward bias $\left(i=I_{\text {sat }}\right)$. Curve a) does not give the $k T / C$ noise of its junction capacitance (see text). 
[7]. The $S_{V S i m}(0)=-155.8 \mathrm{~dB}$ that PSPICE gives at low $f$ for $i=I_{\text {sat }}$ is the shot noise of the net current $i$ converted to voltage noise on $r_{d f w}=62 \mathrm{k} \Omega: 10 \log \left(2 q I_{s a t} \times{r_{d f w}}^{2}\right)=$ $-155.8 \mathrm{~dB}$. This noise is $7.7 \mathrm{~dB}$ below $S_{\text {VAM }}(0)=$ $(3 / 2) 4 k T r_{d f w}=-148.1 \mathrm{~dB}$ that our AM will give for this case out of TE in the next Section. Considering that to deal with shot noise one has to take all the independent currents, not its net value [6], and that a net $i=I_{\text {sat }}$ is: $i=$ $\left(+2 I_{\text {sat }}\right)+\left(-I_{\text {sat }}\right)$, see (3), we have: $S_{I}(f)=2 q\left(I_{\text {sat }}+2 I_{\text {sat }}\right)$ $\mathrm{A}^{2} / \mathrm{Hz}$ that converted to $S_{V}$ on $r_{d f w}$ gives: $10 \log \left(6 q I_{s a t} \times\right.$ $\left.r_{d f w}^{2}\right)=-151 \mathrm{~dB}$. This improvement of the PSPICE's prediction appears "converting" shot noise accordingly to a procedure that works fine in TE, where a net $i=0=$ $\left(+I_{\text {sat }}\right)+\left(-I_{\text {sat }}\right)$ leads to: $S_{I}(f)=2 q\left(I_{\text {sat }}+I_{\text {sat }}\right)$ that converted to $S_{V}$ on $R^{*}$ gives: $S_{V}(0)=10 \log \left[4 q I_{s a t} \times\left(R^{*}\right)^{2}\right]$ (e.g. $S_{V}(0)=-146.8 \mathrm{~dB}$ ) which is similar to $S_{V A M}(0)=$ $4 k T R^{*}=-146.9 \mathrm{~dB}$ predicted by our AM.

As it is shown in $[5,7]$, this "conversion" by the square of a resistance fails out of TE [5,7] as we will see in the next Section linked with the meaning of Resistance in time domain. A proof favouring our AM that doesn't need this advanced knowledge can be given from a paradox we had in previous Section where a negative feedback seemed to improve the $S / N$ of the pixel of Figure 3 accordingly to the PI in use today. Following our $\mathrm{AM}$, however, the $k T / C$ noise of $C$ must be kept by taking the low $f$ noise density on $R^{*}$ as: $S_{V A M}(0)=4 k T R^{*}$, where $R^{*}$ is the resistance shunting $C$ no matter its origin. If $R^{*}=R$ in the pixel without feedback we have: $S_{V A M}(0)$ $=4 \mathrm{kTR} \mathrm{V}^{2} / \mathrm{Hz}$ and when feedback makes $R^{*}=R / 2$, the noise is: $S_{V A M}(0)=4 k T R / 2 \mathrm{~V}^{2} / \mathrm{Hz}$, always keeping the $k T / C$ noise of $C$. This keeps the noise power on $R: N_{F B}=$ $k T /(R C) \mathrm{W}$ and therefore, this negative feedback doesn't improve the $S / N$ as it is "well known".

When $v$ is negative and few times $V_{T}$, an interesting situation appears because the diode current becomes $i \approx$ $-I_{\text {sat }}$ that, accordingly to the PI today in use, is a source of "shot" noise $S_{I}(f) \approx 2 q\left(I_{\text {sat }}\right) \mathrm{A}^{2} / \mathrm{Hz}$ that converted to voltage noise $\left(\mathrm{V}^{2} / \mathrm{Hz}\right)$ by $r_{d}^{2}(v)$ will track the $\exp \left(-v / n V_{T}\right)$ variation of $r_{d}(v)$ for $C_{J 0}(v) \approx$ constant. This is shown in Figure 6(a) by the noise spectra PSPICE gives for the diode biased by three reverse currents $i_{a}, i_{b}$ and $i_{c}$ that set $v_{a}=-3 n V_{T}, v_{b}=-4 n V_{T}$ and $v_{c}=-5 n V_{T}$ in the diode respectively. To study the noise of the diode alone, $R_{1}$ was removed from the PSPICE model.

Due to the factor $e \approx 2.718 \ldots$ dividing $r_{d}(v)$ as we go from curve a) to curve b) of Figure 6(a), the bandwidth decreases by $e$ whereas their low $f$ value increases by $e^{2}$. This gives Figure 6(a) looking like the picture representing the Gain $\times$ Bandwidth conservation of Operational Amplifiers with resistive feedback. The results of our AM for these cases will be discussed in the next Section.

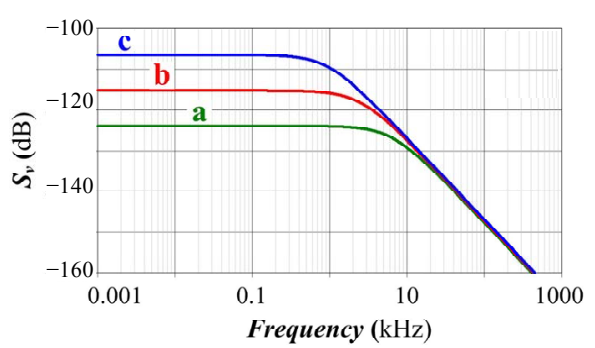

(a)

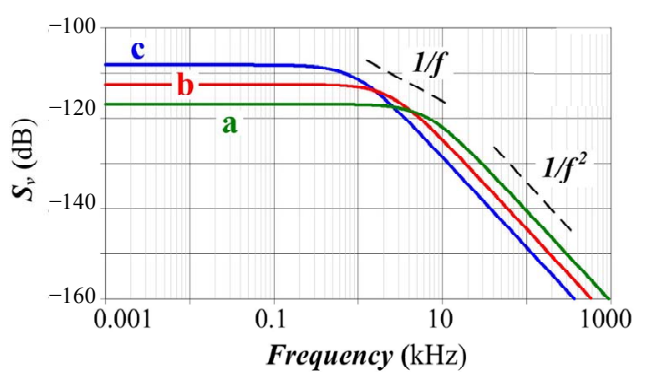

(b)

Figure 6. (a) Noise densities $S_{V}(f)$ obtained by PSPICE for the BAT85 Schottky diode at room $T$ for three reverse currents (see text); (b) Noise densities $S_{V}(f)$ obtained by our Fluctuation-Dissipation model for electrical noise.

\section{Electrical Noise as Fluctuations and Dissipations of Electrical Energy in Time}

Because an arbitrary voltage noise $v(t)$ can be built from sinusoidal components (Fourier synthesis) we will use sinusoidal noise voltages $v_{n}(t)$ and currents $i_{n}(t)$ except otherwise stated. Being Conductance $G(f)$ the ratio between sinusoidal current and voltage mutually in-phase at frequency $f$, it doesn't distinguish "ohmic" resistances of resistors from "non-ohmic" ones due to feedback or to junctions for example, because all of them dissipate power by Conduction Currents (CC) in phase with their voltage. To work with a parallel circuit let's use the Admittance function $Y(j \omega)=G(\omega)+j B(\omega)$, whose Real part is Conductance $G(\omega)$ and whose Imaginary part is Susceptance $B(\omega)$, where $\omega$ is the angular frequency $\omega=2 \pi f$ and $j$ is the imaginary unit indicating a $90^{\circ}$ phase shift (orthogonality or null mean overlap in time for sinusoidal signals).

From the meaning of $Y(j \omega)$, a voltage noise $v_{n}(t)$ observed between terminals of a noisy device will come from a current in-phase with $v_{n}(t)$ through $G=1 / R^{*}$ and from a current in-quadrature through $B(\omega) . G=1 / R^{*}$ means that we will use a Conductance that is independent of $f$ as it uses to happen in the device resistor at low $f$, because at high $f$ it becomes a capacitor due to $\tau_{d}$ [7]. Figure 7 shows the circuit our AM uses for the electrical noise of resistors and capacitors in TE. Although it reminds the parallel one of Figure 2, it has $C=C_{d}+C_{\text {stray }}$ 
shunting its $R^{*}$ that includes ohmic and non-ohmic resistances defining the Conductance $G=1 / R^{*}$ of the Admittance we will use to handle noise voltage $v(t)$ as the signal linking noise current in its Conductance with noise current in its Susceptance at each $f$. Being $v_{n}(t)$ a sinusoidal component of $v(t)$, the noise current through $G=$ $1 / R^{*}$ is the sinusoidal current in-phase with $v_{n}(t)$ given by: $i_{P}(t)=G \times v_{n}(t)$ (see Figure 8).

The noise current $i_{Q}(t)$ of Figure 8 is a noise current in-quadrature with $v_{n}(t)$ given by: $i_{Q}(t)=j B \times v_{n}(t)$ that, for this positive $B$ due to $C$, will be $+90^{\circ}$ phase-advanced respect to $v_{n}(t)$. Hence, the time integral of $i_{Q}(t)$ and $v_{n}(t)$ have the same Phase, thus suggesting what our AM considers: that the Displacement Current (DC) at each $f i_{Q}(t)$ is the Cause that, integrated in time by $\mathrm{C}$, produces the Effect $v_{n}(t)$ that synthesizes Johnson noise in resistors and $k T / C$ noise in capacitors. To study noise coming from Fluctuations in time of electrical energy, let's use the instantaneous power function: $p_{i}(t)=v_{n}(t) \times i_{n}(t)$ where $i_{n}(t)=i_{P}(t)+i_{Q}(t)$ is the whole current in the device. To work in time domain, the noise density $4 k T / R^{*}$ $\mathrm{A}^{2} / \mathrm{Hz}$ of Figure 7 is replaced by the random current $i_{N y}(t)$ with zero mean shown in Figure 8, whose equation on top (Kirchoff's law) we will write as:

$$
C \frac{\partial v_{n}(t)}{\partial t}=-G v_{n}(t)+i_{N y}(t)
$$

Equation (4) states that any real $i_{N y}(t)$ will create both a DC in $C$ and a CC in $R^{*}$. To create only a DC, a $\delta$-like $i_{N y}(t)$ of infinite bandwidth $(\mathrm{BW} \rightarrow \infty)$ or null duration ( $\delta t_{c} \rightarrow 0$ ) is needed. For this $\delta$-like $i_{N y}(t)$ having a weight $q$ in $t$ (charge), the time integral of (4) during $\delta t_{c} \rightarrow 0$ gives a step $\Delta v_{\text {built }}=q / C \mathrm{~V}$ created instantaneously in $C$ by the $\delta$-like $i_{N y}(t)$.

For an uncharged $C(v=0)$, a Fluctuation of $q^{2} /(2 C) J$ appears in $C$, whence it may be seen the need of $C$ to have fluctuations of electrical energy in a resistor. Although pure Fluctuations of energy won't exist in resistors because their $i_{N y}(t)$ is band-limited [10], there can be fast DC with short $\delta t_{c}$ looking like very pure Fluctuations of energy in this circuit. They will include, however, a small, non null Dissipation due to the CC existing during the non null $\delta t_{c}$ elapsed, whence it may be seen that an electron leaving one plate with a kinetic energy of $q^{2} /(2 C) J$ exactly is unable to create $\Delta v_{\text {built }}=q / C \mathrm{~V}$ in $C$ because part of its $q^{2} /(2 C)$ energy is dissipated during its transit time $\delta t_{c}$. For $\delta t_{c} \ll R^{*} C$ however, this passage of one electron between plates of $C$ is a very energyconserving event because the term $G v_{n}(t)$ "has no time" to dissipate a noticeable energy during this fast DC or thermal jump of an electron of charge $q$ between the ohmic contacts or plates of $C$. This fast DC would be a pure Fluctuation of energy (e.g. devoid of Dissipation) given the orthogonal character of these two phenomena (see Appendix II).

Let's call Thermal Action (TA) this fast transit of an electron between the ohmic contacts due to thermal activity. For a capacitor made from two metal plates in vacuum, thermoionic emission would produce directly these TA [5], whereas for resistors with conducting material between plates, TA would appear in the way proposed in Appendix III. Once a TA sets a voltage $\Delta v_{\text {built }}(t$ $=0)=q / C$ in $C$, the response of the circuit to this impulsive driving starts. This response or Reaction is a slower CC driven by $\Delta v_{\text {built }}(t)$ itself that decays exponentially with $t$ as the Fluctuation of energy (Cause) is dissipated by the CC or Effect it produces. Thus, this Reaction includes a CC through $G$ together with a simultaneous DC through $C$ to rearrange the dipolar charge $\Delta Q(t)=C \times$ $\Delta v_{\text {built }}(t)$ that sustains $\Delta v_{\text {built }}(t)$ at each instant of $t$. Making null $i_{N y}(t)$ in (4) or in Figure 8, the continuity of electrical current states that this exponentially decaying $\mathrm{CC}$ in $G$ requires a similar DC in $C$ as $C$ loses the energy fluctuation it received from the previous TA.

Calling this process Device Reaction (DR) electrical noise becomes a random series of Action-Reaction events taking place in an electrical Admittance. This explains and allows an easy simulation of Phase Noise in oscillators based on $L-C$ resonators $[16,17]$ for example. This description in time of the noise densities $S_{I}(f)$ and $S_{V}(f)$ considers TA appearing randomly, both in time and sign, at an average rate of $\lambda$ TA per second to present Conductance $\mathrm{G}=1 / R$ as a rate of chances to dissipate energy accordingly to (9) in Appendix III, where we have considered these fast DC are carried by electrons independently of one another as Johnson did in 1925 [2].

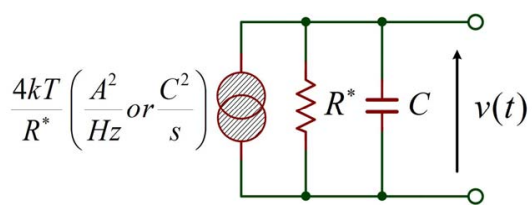

Figure 7. Electrical circuit that represents Johnson noise of resistors or $k T / C$ noise of capacitors in Thermal Equilibrium accordingly to our Fluctuation-Dissipation Model for electrical noise.

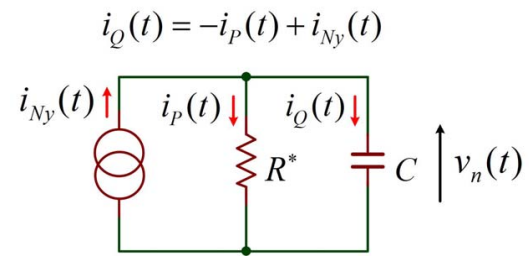

Figure 8. Time-domain counterpart of the Fluctuation-Dissipation Model of Figure 7 used to show the Cause-Effect connection of the Fluctuation-Dissipation pair of events. 
This view of $G$ as $\lambda$ chances per second to dissipate energy suggests the reason why the conversion $\mathrm{A}^{2} / \mathrm{Hz} \rightarrow$ $\mathrm{V}^{2} / \mathrm{Hz}$ by the square of a resistance fails for very high $R^{*}$ values, as those found in [7] for the $r_{d}(v)$ of reverse-biased junctions with tens of $V_{T}$. The high noise voltages this conversion gives as $R^{*} \rightarrow \infty$ would require "packets of electrons" passing together between terminals [5] that, contrarily to [3], we consider unlikely to occur in common devices. This led us to abandon this conversion in [7] replacing it by the AM or Fluctuation-Dissipation Model (FDM) for electrical noise just described, which was used to explain two noises [5,7] that the PI of Nyquist result in use today is unable to explain.

To use this FDM out of TE let's consider the cases of the BAT85 diode whose PSPICE spectra appeared in Figure 6(a). Recalling that for $v=0$ the diode is in TE with $\lambda \mathrm{TA} / \mathrm{s}$ due to its $t w o$ opposed $I_{\text {sat }}$, the rate of $T A$ for $i \approx-I_{\text {sat }}$, will drop to $\lambda / 2[5,7]$. Since $C$ only has half the charge noise power it had in TE, we don't have to keep $\left\langle v^{2}(t)\right\rangle=k T / C$ in $C$, but half this value. Particularizing (2) for these cases, we have:

$$
\begin{aligned}
S_{\text {VAMOUT }}(f) & =\frac{2 k \operatorname{Tr}_{d}(v)}{1+\left(2 \pi f r_{d}(v) C_{J 0}(v)\right)^{2}} \\
& =\frac{k T}{C_{J 0}(v)} \times \frac{1}{\pi} \times \frac{\frac{1}{f_{c}(v)}}{1+\left(\frac{f}{f_{c}(v)}\right)^{2}}
\end{aligned}
$$

Integrating (5) from $f \rightarrow 0$ to $f \rightarrow \infty$ we obtain: $\left\langle v^{2}(t)\right\rangle=k T /\left[2 C_{J 0}(v)\right]$. Figure 6(b) shows the three noise spectra obtained using (5) with the $f_{c}$ PSPICE gave in Figure 6(a). They form a ladder of slope $1 / f$ whose sum gives a $1 / f$ noise spectrum over a band $\left(f_{L O}-f_{H I}\right)$ such that: $f_{I O} / f_{H I}=\exp \left[\left(5 n V_{T}-3 n V_{T}\right) / V_{T}\right]$. This means one decade of $1 / f$ noise for a voltage span of $2.3 V_{T}$ (e.g. $60 \mathrm{mV}$ at room $T$ ), being this the basis of the $1 / f$ noise synthesizer that appeared in [5,7] with this FDM for electrical noise based on Admittance.

To end, let's justify the $S_{V A M}(0)=(3 / 2) 4 k T r_{d f w}=$ $-148.1 \mathrm{~dB}$ value we proposed in previous Section as the noise for the diode with $i=I_{\text {sat }}$, recall curve b) of Figure 5. Due to the $-I_{\text {sat }}$ and $+2 I_{\text {sat }}$ existing in this case, we have: $3(\lambda / 2) \mathrm{TA} / \mathrm{s}$ in $C$ or a charge noise power that is $3 / 2$ times higher than the one required to keep the $k T / C$ noise of $C$ by $\lambda \mathrm{TA} / \mathrm{s}$ in TE. This means that for $i$ $=I_{\text {sat }}$ we don't have to keep $\left\langle v^{2}(t)\right\rangle=k T / C \mathrm{~V}^{2}$ in $C$ by using $4 k T r_{d f w} \mathrm{~V}^{2} / \mathrm{Hz}$ as the low $f$ value of (2). Instead, we have to keep $3 / 2$ times this value, whence it can be seen the reason to write $S_{V A M}(0)=(3 / 2) 4 k T r_{d f w}$ $=6 k T r_{d f w}$.

\section{Conclusions}

The partial interpretation of Nyquist result today in use leads to a 1-Dimensional (1-D) noise model based on Dissipation that is incomplete. A 2-D noise model using a Complex Admittance to handle Fluctuation and Dissipation of electrical energy, not only excels the aforesaid 1-D model, but also studies electrical noise accordingly to its first Quantum Mechanical treatment due to Callen and Welton. In this Complex model, Fluctuations and Dissipations of electrical energy creating electrical noise form a random series of Cause/Effect pairs in time, each linked with an elemental charge noise of one electron.

For this Complex model of electrical noise, $1 / f$ "excess noise" in Solid State devices and flicker noise in thermoionic emitters simply are consequences of thermal noise that the 1-D noise model based on Dissipation is unable to explain. This Complex model also shows that the Johnson noise of resistors and the $k T / C$ noise of capacitors, both reflect the same power $4 k T / R$ of charge noise in $\mathrm{C}^{2} / \mathrm{s}$ or $\mathrm{A}^{2} / \mathrm{Hz}$, appearing thermally between two conductors separated by some finite distance in our physical world. The quantization of electrical noise that results leads to an easy explanation of Phase noise in resonant circuits of electronic oscillators.

\section{References}

[1] L. Callegaro, "Unified Derivation of Johnson and Shot Noise Expressions," American Journal of Physics, Vol. 74, No. 5, 2006, pp. 438-440. doi:10.1119/1.2174034

[2] J. B. Johnson, "The Schottky Effect in Low Frequency Circuits," Physical Review, Vol. 26, No. 1, 1925, pp. 71-85. doi:10.1103/PhysRev.26.71

[3] G. Gomila, C. Pennetta, L. Reggiani, M. Sampietro, G. Ferrari and G. Bertuccio, "Shot Noise in Linear Macroscopic Resistors," Physical Review Letters, Vol. 92, 2004, Article ID: 226601. doi:10.1103/PhysRevLett.92.226601

[4] H. B. Callen and T. A. Welton, "Irreversibility and Generalized Noise," Physical Review, Vol. 83, No. 1, 1951, pp. 34-40. doi:10.1103/PhysRev.83.34

[5] J. I. Izpura, "On the Electrical Origin of Flicker Noise in Vacuum Devices," IEEE Transactions on Instrumentation and Measurement, Vol. 58, No. 10, 2009, pp. 3592-3601. doi:10.1109/TIM.2009.2018692

[6] J. I. Izpura and J. Malo, "Noise Tunability in Planar Junction Diodes: Theory, Experiment and Additional Support by SPICE," TAEE'2006 Conference, Madrid, July 2006. http://taee.euitt.upm.es/Congresosv2/2006/papers/2006S1 F02.pdf

[7] J. I. Izpura, "1/f Electrical Noise in Planar Resistors: The Joint Effect of a Backgating Noise and an Instrumental Disturbance," IEEE Transactions on Instrumentation and Measurement, Vol. 57, No. 3, 2008, pp. 509-517. doi:10.1109/TIM.2007.911642 
[8] M. Sampietro, L. Fasoli and G. Ferrari, "Spectrum Analyzer with Noise Reduction by Cross-Correlation Technique on Two Channels," Review of Scientific Instrumentation, Vol. 70, No. 5, 1999, pp. 2520-2525. doi:10.1063/1.1149785

[9] J. B. Johnson, "Thermal Agitation of Electricity in Conductors," Physical Review, Vol. 32, No. 1, 1928, pp. 97-109. doi:10.1103/PhysRev.32.97

[10] H. Nyquist, "Thermal Agitation of Electric Charge in Conductor," Physical Review, Vol. 32, No. 1, 1928, pp. 110-113. doi:10.1103/PhysRev.32.110

[11] D. A. Johns and K. Martin, "Analog Integrated Cicuit Design,” John Wiley \& Sons, New York, 1997.

[12] "Dual Low Noise, Picoampere Bias Current, JFET Input Op Amp,"

http://www.datasheetcatalog.org/datasheet/lineartechnolo

\section{Appendix I}

\section{SUBCKT BAT85 12}

* The Resistor R1 does not reflect a physical device. Instead it improves modelling in the reverse mode of operation.

R1 $123.6 \mathrm{E}+07$

D1 12 BAT85-1

.ENDS

*

.MODEL BAT85-1 D(IS $=2.117 \mathrm{E}-07, \mathrm{~N}=1.016, \mathrm{BV}$ $=36, \mathrm{IBV}=1.196 \mathrm{E}-06, \mathrm{RS}=2.637, \mathrm{CJO}=1.114 \mathrm{E}-11$, $\mathrm{VJ}=0.2013, \mathrm{M}=0.3868, \mathrm{FC}=0, \mathrm{TT}=0, \mathrm{EG}=0.69$, $\mathrm{XTI}=2$ )

A similar model can be found in:

http://www.nxp.com/models/spicespar/BAT85.html

\section{Appendix II}

For a sinusoidal $v_{n}(t)$ in the circuit of Figure 8 the current $i_{P}(t)$ also is sinusoidal whereas $i_{Q}(t)$ is advanced $+90^{\circ}$ respect to $v_{n}(t)$. Thus, the instantaneous power contains Active power $p_{i P}(t)=v_{n}(t) \times i_{P}(t)$ always entering $R^{*}$ and Reactive power $p_{i Q}(t)=v_{n}(t) \times i_{Q}(t)$ entering and leaving the Susceptance (e.g. oscillating) at $2 f$. Taking $v_{n}(t)=A \times \sin (\omega t)(\omega=2 \pi f)$ as a voltage existing on $C$ and from its related current in $C: i_{Q}(t)=C \times\left(\partial v_{n}(t) / \partial t\right)$, the instantaneous power $p_{i Q}(t)$ entering $C$ is:

$$
\begin{aligned}
p_{i Q}(t) & =A \sin (\omega t) \times(A \omega C) \cos (\omega t) \\
& =\left(A^{2} \omega C\right) \times \sin (\omega t) \times \cos (\omega t)
\end{aligned}
$$

On the other hand, the fluctuating electrical energy stored in $C$ by $v_{n}(t)$ will be:

$$
\text { gy/1169fa.pdf }
$$

[13] C. D. Motchenbacher and F. C. Fitchen, "Low Noise Electronic Design," John Wiley \& Sons, New York, 1973.

[14] D. A. Bell, "Noise and the Solid State," Pentech Press, London, 1985.

[15] J. Malo and J. I. Izpura, "Feedback-Induced Phase Noise in Microcantilever-Based Oscillators," Sensors and Actuators A: Physical, Vol. 155, No. 1, 2009, pp. 188-194. doi:10.1016/j.sna.2009.08.001

[16] J. Malo and J. I. Izpura, "Feedback-Induced Phase Noise in Resonator-Based Oscillators," Proceedings of DCIS'09 Conference, Zaragoza, November 2009, pp. 231-236. http://www.linkpdf.com/ebook-viewer.php?url=http://dci s2009.unizar.es/FILES/CR2/p5.pdf

[17] J. Malo, PhD Thesis, Chapter III (in Spanish). Universidad Politecnica de Madrid, Madrid, pp. 70-145.

$$
\begin{aligned}
& U_{E}(t)=\frac{C}{2} \times(A \sin (\omega t))^{2} \Rightarrow \\
& \frac{\partial U_{E}}{\partial t}=\left(A^{2} \omega C\right) \times \sin (\omega t) \times \cos (\omega t)
\end{aligned}
$$

From (6) and (7) all the power entering $C$ leads to fluctuations of its energy, thus linking Fluctuations of electrical energy with currents in quadrature with $v_{n}(t)$. Concerning Dissipations of electrical energy, they come from currents in-phase with $v_{n}(t)$. From the $i_{P}(t)=$ $v_{n}(t) / R^{*}$ that $v_{n}(t)$ produces in the resistance $R^{*}$, the instantaneous power $p_{i P}(t)$ entering $R^{*}$ is:

$$
\begin{aligned}
p_{i P}(t) & =A \sin (\omega t) \times \frac{A}{R^{*}} \sin (\omega t) \\
& =\frac{A^{2}}{2 R^{*}}(1-\cos (2 \omega t))
\end{aligned}
$$

Contrarily to (6) whose mean value is null because energy enters and exits $C$, energy always enters $R^{*}$ and (8) is the power dissipated in a resistance $R^{*}$ driven by sinusoidal voltage of amplitude $A \mathrm{~V}$ : a positive power of mean value $P_{\text {avg }}=A^{2} /\left(2 R^{*}\right) \mathrm{W}$. Thus all the instantaneous power entering $R^{*}$ is dissipated. Hence, Fluctuation of electrical energy in $C$ and Dissipation of electrical energy in $R^{*}$ are orthogonal processes linked with the Active and Reactive power in the circuit of Figure 8.

\section{Appendix III}

The idea of electrical noise as due to a random series of Fluctuation-Dissipation events or TA-DR pairs taking place in the Admittance of Figure 8 leads to consider the evolution of $\Delta v_{\text {built }}=q / C \mathrm{~V}$ created by each TA in $C$. Being the sign of $\Delta v_{\text {built }}$ positive or negative with equal 
probability on average, the mean voltage in $C$ will be null $(v=0)$. Taking $\lambda / 2$ as the average rate of positive $\mathrm{TA}$, the average rate of TA is $\lambda$. For $v=0$, each TA or Charge fluctuation of one electron in $C$ will set a Fluctuation $\Delta U_{T A}=q^{2} /(2 C) \mathrm{J}$ in $C$. Once this Fluctuation is completed in a short transit time $\tau_{T A}$, its associated voltage $\Delta v_{\text {built }}=q / C$ starts to drive a slower DR or the Dissipation by $R^{*}$ of the $\Delta U_{T A}$ stored by the TA. Due to this, the initial $\Delta v_{\text {built }}$ decays with a time constant $\tau=R^{*} C$ as shown in Figure 9(a). The spectrum of this decay will look like the Lorentzian one of Figure 9(b), which is the Johnson noise $v(t)$ of the circuit of Figure 7 coming from a random series of pulses like that of Figure 9(a), each following its own TA (Carson's Theorem).

Since the noise power $N$ dissipated in $R^{*}$ (the $k T / C$ noise of $C$ divided by $R^{*}$ ) has to dissipate the energy fluctuations of $\lambda$ TAs per second on average, we have:

$$
N=\frac{k T}{R^{*} C}=\lambda \times \frac{q^{2}}{2 C} \Rightarrow R^{*}=\frac{2 k T}{q^{2} \lambda} \Rightarrow \frac{4 k T}{R^{*}}=2 q(\lambda q)
$$

Equation (9) shows that the Resistance $R^{*}$ of a resistor or capacitor in TE is inversely proportional to the $\lambda$ opportunities per unit time it has to dissipate energy. It also shows that the noise density $4 k T / R^{*} \mathrm{~A}^{2} / \mathrm{Hz}$ preserving the $k T / C$ noise of $C$ is the density of shot noise due to the $\lambda$ TA's per unit time taking place in this device.

Thus, the shot noise $2 q I_{T A} \mathrm{~A}^{2} / \mathrm{Hz}$ of the current $I_{T A}=$ $\lambda q$ associated to the $\lambda$ TAs per unit time of this device is the source of its electrical noise, no matter if we call it Johnson noise of its resistance $R^{*}$ or $k T / C$ noise of its capacitance $C$. This deep connection between "shot" and Johnson noises (also found by other scientists [1]) is the charge noise existing in the capacitance $C$ of a resistor or capacitor in TE due to its $\lambda$ TA-DR pairs per unit time that are shown in Figure 10.

Since each DR has a $\mathrm{CC}$ and a $\mathrm{DC}$ giving a charge fluctuation of $q \mathrm{C}$ each in $C$ and they are uncorrelated in time due to their orthogonal character in $f$-domain, the mean square charge noise per second (charge noise power) existing in $C$ due to DR is: $2\left(\lambda q^{2}\right)=4 k T / R^{*} \mathrm{C}^{2} / \mathrm{s}$. This explains the alternative units used in Figure 7 to reveal neatly the same charge noise underlying Johnson and shot "noises".

It is worth studying what happens if we change the conducting material between plates in Figure 1 to reduce its $R^{*}$ to $R^{*} / 10$ while keeping the same $\varepsilon$. Since $C$ does not vary its $k T / C$ noise will remain, but the voltage decays of Figure 9(a) will be ten times faster, thus having a ten times broader spectrum (Figure 9(b)). Since the voltage $\Delta v_{\text {built }}=q / C$ decays ten times faster with $t$, there must be ten times more TA $(10 \lambda)$ to sustain the same $k T / C \mathrm{~V}^{2}$ in time. This agrees with (9), where 10 times lower $R^{*}$ at the same $T$ requires a 10 times higher rate of TA or 10 times more charge noise due to TA. Since this happens no matter the material we use, the presence of solid matter between contacts doesn't change the nature of the TA in our FDM: a Charge Noise of one electron.

Although single electrons jumping between plates in vacuum is well know [2], this is not so for electrons doing it between contacts in Solid-State devices due to their possible blockage by the conducting material between them. This is solved, however, by considering that a free electron in a Quantum State (QS) of the Conduction Band (CB) has a wavefunction extending over the whole material between plates. This would allow an electron to jump from a contact to this QS as soon as it is left empty by the electron that, occupying it previously, has been captured by the far contact (Collector). Thus, a QS or energy level of the CB would be a sort of tunnel for the fast transit of each electron between ohmic contacts cladding n-type semiconductor material for example.

Used to electrons transiting between electrodes in vacuum devices that are collected a short transit time $\tau_{T}$ after their emission, the above process looks reversed: an electron of the $\mathrm{CB}$ is captured by the Collector a short time $\tau_{T}$ before another electron is emitted from the Emitter contact to the empty QS of the CB. Processes where electrons emitted from a contact to empty QS of the CB are subsequently captured by the other contact acting as a Collector of electrons are equally possible.

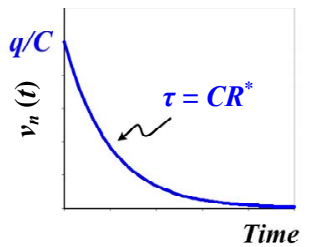

(a)

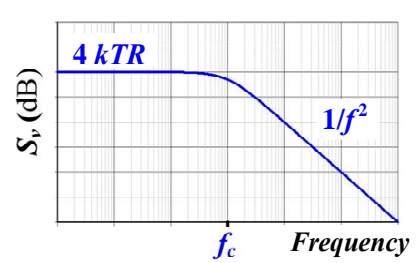

(b)
Figure 9. (a) Impulse response of the circuit of Figure 8 driven by current $i_{N y}(t)$ and taking $v(t)$ as its output signal. (b) Bode plot (modulus) of the Lorentzian spectrum of $v(t)$ mirroring the spectral energy content of the impulse shown in Figure 4.

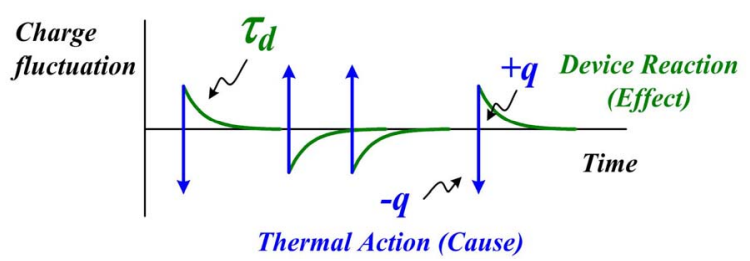

Figure 10. Charge noises in the contacts of a resistor or capacitor due to the (Thermal Action)/(Device Reaction) dynamics described in the text. 